\title{
BMJ Open Randomised controlled trial of online continuing education for health professionals to improve the management of chronic fatigue syndrome: a study protocol
}

\author{
Sophie H Li, ${ }^{1,2}$ Carolina X Sandler, ${ }^{1,3}$ Sally M Casson, ${ }^{1}$ Joanne Cassar, ${ }^{1,3}$ \\ Tina Bogg, ${ }^{1}$ Andrew R Lloyd, ${ }^{3}$ Benjamin K Barry ${ }^{1,4,5}$
}

To cite: Li SH, Sandler CX, Casson SM, et al. Randomised controlled trial of online continuing education for health professionals to improve the management of chronic fatigue syndrome: a study protocol. BMJ Open 2017;7:e014133. doi:10.1136/ bmjopen-2016-014133

- Prepublication history and additional material are available. To view these files please visit the journal online (http://dx.doi.org/ 10.1136/ bmjopen-2016-014133)

Received 2 September 2016 Revised 7 March 2017 Accepted 13 March 2017

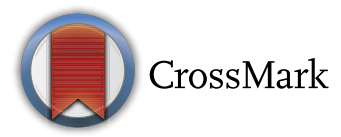

${ }^{1}$ School of Medical Sciences, University of New South Wales, Sydney, Australia

${ }^{2}$ School of Psychology, The University of New South Wales, Sydney, Australia

${ }^{3}$ The Kirby Institute, University of New South Wales, Sydney, Australia

${ }^{4}$ Neuroscience Research Australia, University of New South Wales, Sydney, Australia ${ }^{5}$ School of Clinical Medicine, University of Queensland, Brisbane, Australia

\section{ABSTRACT}

Introduction Chronic fatigue syndrome (CFS) is a serious and debilitating illness that affects between $0.2 \%-2.6 \%$ of the world's population. Although there is level 1 evidence of the benefit of cognitive behaviour therapy (CBT) and graded exercise therapy (GET) for some people with CFS, uptake of these interventions is low or at best untimely. This can be partly attributed to poor clinician awareness and knowledge of CFS and related CBT and GET interventions. This trial aims to evaluate the effect of participation in an online education programme, compared with a wait-list control group, on allied health professionals' knowledge about evidence-based CFS interventions and their levels of confidence to engage in the dissemination of these interventions.

Methods and analysis A randomised controlled trial consisting of 180 consenting allied health professionals will be conducted. Participants will be randomised into an intervention group $(n=90)$ that will receive access to the online education programme, or a wait-list control group $(n=90)$. The primary outcomes will be: 1) knowledge and clinical reasoning skills regarding CFS and its management, measured at baseline, postintervention and follow-up, and 2) self-reported confidence in knowledge and clinical reasoning skills related to CFS. Secondary outcomes include retention of knowledge and satisfaction with the online education programme. The influence of the education programme on clinical practice behaviour, and self-reported success in the management of people with CFS, will also be assessed in a cohort study design with participants from the intervention and control groups combined.

Ethics and dissemination The study protocol has been approved by the Human Research Ethics Committee at The University of New South Wales (approval number HC16419). Results will be disseminated via peer-reviewed journal articles and presentations at scientific conferences and meetings. Trial registration ACTRN12616000296437.

\section{Strengths and limitations of this study}

- Randomised controlled trial.

- Objective and subjective assessment measures.

- Translation of evidence-based interventions to practice.

- Engaging multiple allied health professions.

- Follow-up measurements made on a cohort rather than on intervention and control groups.

- Actual impact on practice is assessed only by clinician report and in the short term.

- Trial recruitment is confined to Australia.

- Customised assessments for this study have not previously been validated.

\section{INTRODUCTION}

Chronic fatigue syndrome (CFS) refers to the presence of persistent and severe fatigue that is accompanied by musculoskeletal pain, neurocognitive difficulties, in addition to sleep and mood disturbances, and cannot be accounted for by a medical condition. ${ }^{1}$ It is a serious and debilitating illness that affects between $0.2 \%$ $2.6 \%$ of the world's population. ${ }^{2}$ Cochrane reviews of both cognitive behaviour therapy $(\mathrm{CBT})^{3}$ and graded exercise therapy (GET) ${ }^{4}$ provide level 1 evidence of their benefits for people with CFS. More than 20 randomised controlled trials by independent researchers examining the effectiveness of CBT or GET across separate patient groups and in various geographical locations have found moderately beneficial effects of these interventions for CFS, including significantly reduced levels of fatigue, functional impairment, depression and anxiety, in groupwise outcome analyses (for a review see reference 5 ). ${ }^{5}$ There is also recent evidence that combining $\mathrm{CBT}$ and GET may be more effective than CBT alone. ${ }^{6}$ When applied appropriately the interventions 
are not associated with harm, ${ }^{478}$ and the beneficial effects vary in magnitude from negligible to clinically significant $^{34}$ (This conclusion relates to patients who are able to attend a clinic and may not generalise to more disabled patients). These interventions have also proved generally effective in routine clinical practice. ${ }^{9}$ In the absence of a clear understanding of the underlying pathophysiology of CFS, or curative treatments, as these are the only evidencebased interventions aimed at managing symptoms and improving function, they should be readily available. ${ }^{10}$ Yet uptake of evidence-based CFS management programmes delivered by allied health professionals such as psychologists, exercise physiologists and physiotherapists is low. ${ }^{112}$ Recent studies have shown that gaps between research and practice are at least partially due to allied health professionals lacking the knowledge and skills to provide appropriate care, ${ }^{13}{ }^{14}$ and potentially also the effects of the controversy regarding the PACE (Pacing, graded Activity, and Cognitive behaviour therapy; a randomised Evaluation) trial analysis. ${ }^{15}$

For several reasons it is crucial that allied health professionals have the capacity to provide evidence-based CBT and GET interventions to patients with CFS. First, it should be a patient's right that interventions proven to assist in the management of their symptoms are readily available. Second, it is widely acknowledged that overexertion can lead to exacerbation of fatigue symptoms (for examples see references 16 and 17), ${ }^{16} 17$ thus inappropriate prescription of physical and cognitive activities will result in poor treatment outcome. Many patients and allied health professionals incorrectly assume GET involves the development of a traditional exercise programme (this is an easy mistake to make given the potentially misleading term: GET) that requires marked physical exertion, which should be continued regardless of increasing symptoms. However, after appropriate day-to-day pacing of regular activities has been established-that is, establishing a daily or weekly schedule of activities that does not exceed the individual's energy thresholds ${ }^{7-}$ GET merely commences with conservative incremental increases in the duration of daily physical activities, including incidental tasks such as domestic chores. This is followed by gradual progression to more structured exercise, such as walking, which is introduced and cautiously increased in a graded fashion, generally at levels far below physical activity guidelines for the general population. ${ }^{7}$ Thus, to provide safe and effective GET for people with CFS some clinicians may need to recalibrate their notions of exercise. The implementation of GET as part of an intervention for people with CFS subsequent to the establishment of appropriate pacing of activities is important because activity pacing alone does not consistently provide benefit. ${ }^{718}$

Finally, and very importantly, many health professionals have outdated views on the aetiology of CFS, which can result in the use of inappropriate interventions and hence patient frustration. Rooted in historically dismissive views from some clinicians is a concern of some people with CFS that the advocacy of a psychological intervention, such as CBT, implies that their illness is 'all in their head' and not a real disease. Education of clinicians about CFS and the role of CBT is an important step to help address such misunderstandings.

Improving allied health professional knowledge via online continuing education activities has been widely documented. ${ }^{1920}$ Yet there has been very little research into interventions that aim to influence allied health professional knowledge and practice in relation to CFS. One study investigated whether community healthcare centres were able to implement and sustain a CBT intervention for people with CFS by using an implementation manual. ${ }^{21}$ They found that while patients showed an improvement in fatigue severity and physical function, health professionals required considerable external support from the authors of the study for successful implementation of the manual. Online education programmes are an alternative method to improve health professional knowledge and practice, as has been demonstrated in other areas of healthcare (eg, references 22 and 23). ${ }^{22}{ }^{23}$ While the impact of online educational interventions has been investigated in some areas of healthcare (eg, references 22-24), ${ }^{22-24}$ none, to our knowledge, have investigated the impact of online educational activities on CFS management. The aim of this trial is to evaluate the effect of participation in an online education programme on allied health professionals' knowledge about evidence-based CFS interventions and their levels of confidence to deliver these interventions, compared with a wait-list control group. Satisfaction with the online education programme will also be assessed, as will retention of knowledge, for the intervention group only. The influence of the education programme on clinical practice behaviour will be assessed in a cohort study design with participants pooled from the intervention and waitlist control groups.

\section{METHODS AND ANALYSIS}

\section{Trial design}

A randomised controlled trial will be conducted in accordance with the Consolidated Standards of Reporting Trials (CONSORT) statement ${ }^{25}$ as shown in figure 1 . The trial design was developed and is reported according to the Recommendations for Interventional Trials (Standard Protocol Items: Recommendations for Interventional Trials; SPIRIT) statement ${ }^{26}$ and the education intervention is described according to the Template for Intervention Description and Replication checklist. ${ }^{27}$ In addition to the randomised controlled trial (RCT), a cohort study will be conducted that will assess changes in self-reported success in the management of people with CFS and practice behaviours from baseline to follow-up for both groups combined.

\section{Eligibility criteria}

Australian allied health professionals (eg, psychologists, exercise physiologists, physiotherapists, occupational 


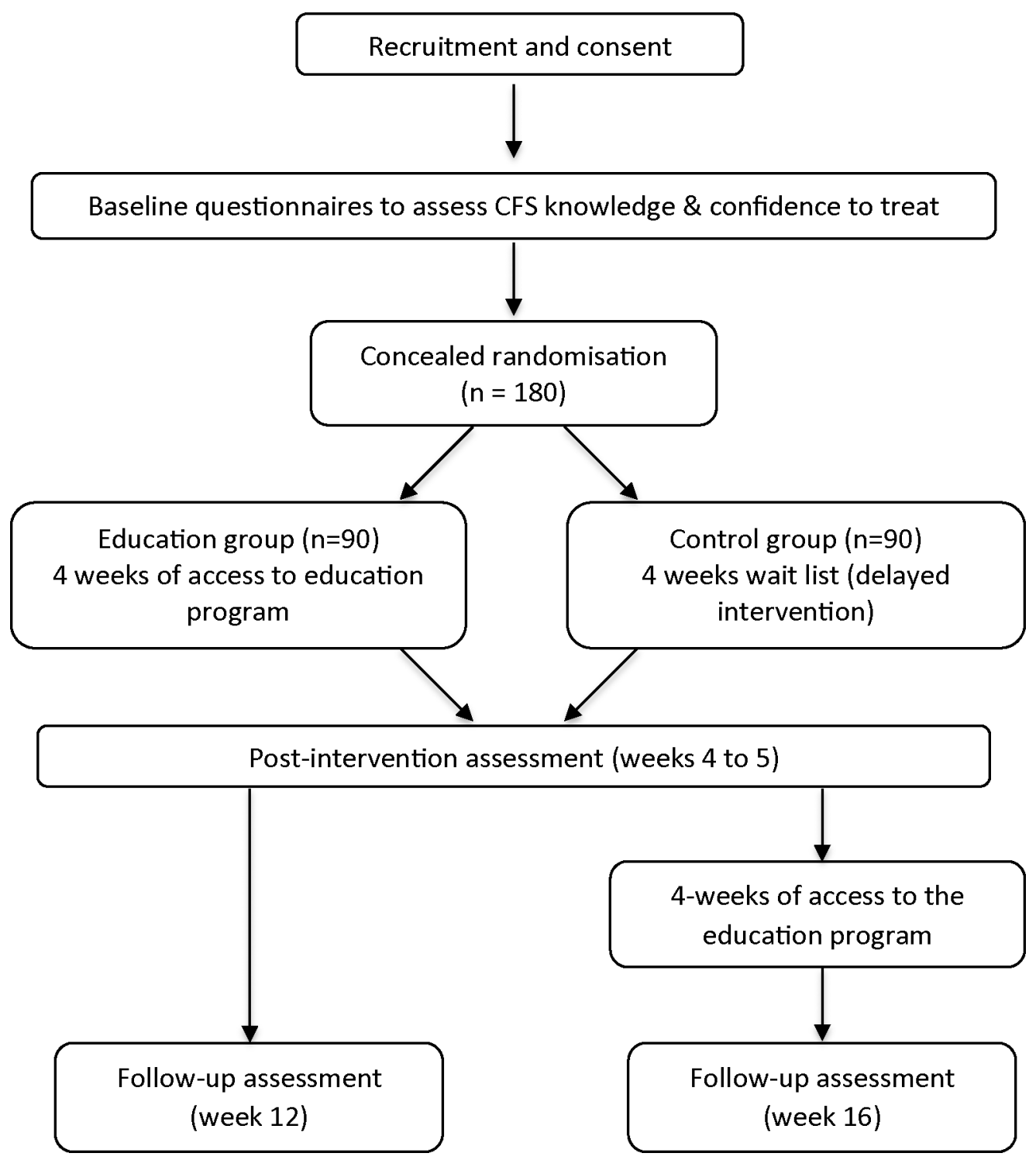

Figure 1 Trial design.

therapists) will be randomly allocated to either the education group (immediate access to the online education programme) or to a wait-list control group. People will be eligible for inclusion in the trial if they are a fully registered, allied health professional. Individuals who are not currently practising will be excluded.

\section{Recruitment}

One hundred and eighty allied health professionals will be recruited via advertisements published in continuing professional development (CPD) calendars or newsletters of professional organisations, or distributed to existing mailing lists of appropriate organisations (eg, Exercise and Sports Science Australia, Australian Physiotherapy Association, Australian Psychology Society, Australian Clinical Psychology Association, Occupational Therapy Australia). The recruitment notices and advertisements will contain a hyperlink that when accessed will provide information about the study and allow the individual to provide consent if wishing to participate. Those consenting to participate will then be contacted by an experimenter with further instructions regarding the trial. On entering the study, the participant is allocated a participant identification code. To protect the participants' privacy, the outcome data are kept in a separate password-protected file from the document containing the participants' names and identification codes. All documents related to the study are stored on a restricted-access server in password-protected files as per University of New South Wales HREC requirements.

Participant recruitment will start in September 2016.

\section{Sample size calculation}

A total sample of 128 participants is required to detect a moderate effect size $(d=0.5)$ of improvement in clinician knowledge of CFS management and confidence to implement evidence-based CFS interventions, with 
a power of $80 \%$ and a two-tailed $\alpha$ of 0.05 . To account for a potential attrition rate of $30 \%$ we intend to recruit 180 participants. These estimates of attrition rate and effect size are based on a similar previous study investigating online education for health professionals. ${ }^{22}$

\section{Randomisation}

Participants will be randomly allocated to the education or wait-list control group after consenting to participate. Randomisation will be conducted by an investigator not involved in recruitment or analysis of data to ensure concealment of allocation. A computer-generated random number sequence with randomly permuted block sizes of two to six will be used to ensure a balance between groups while different allied health professions are successively enrolled.

\section{Intervention}

The online education programme is an interactive, selfpaced online activity that has been developed from a CFS treatment manual previously created, and tested for efficacy, by the research group. ${ }^{7}$ The content contains an introduction and seven modules that include information on CFS symptoms, assessment and diagnosis and detailed descriptions of interventions used to manage CFS symptoms, as well as material on conditions that may occur secondary to fatigue (eg, reduced mood, anxiety, etc). The remaining modules cover: psychoeducation, activity pacing and GET, interventions for sleep disturbance, interventions for cognitive disturbance (eg, cognitive remediation), and interventions for mood, anxiety and emotion coping. Intervention adherence will be tracked automatically by using the online platform to provide data on total time spent on the whole intervention, as well as time spent on each module, and on integrated assessment tasks and other facets of the online education programme. To reduce contamination each participant has an individual password to access the online education programme and are asked not to share this access by providing their password to others. They must also be enrolled into the programme by the experimenter (and are subsequently unenrolled once they have received 4 weeks access to the programme). Furthermore, feedback regarding correct responses to the outcome measures (ie, the multiple choice questions (MCQs) and case vignettes) is not provided, therefore cannot be shared between participants (table 1).

\section{Control group}

The wait-list control group will be given access to the online education programme immediately after completing the postintervention assessment measures, which will be $4-5$ weeks after completion of the baseline assessment measures.

\section{Outcomes}

All outcomes (with the exception of adherence to and satisfaction with the online education activity) will be determined by participants completing an online questionnaire and assessment at three time points. Both groups will complete the outcome measures at baseline (week 0) and postintervention (weeks 4-5), while the follow-up measures will be completed in week 12 for the education group and week 16 for the wait-list control group (ie, 8 weeks after cessation of access to the online education for both groups). Adherence to and satisfaction with the online education programme will be collected postassessment for the education group only. In addition, information regarding profession type of the individual and years of practice will be collected to determine profession and level of professional experience.

\section{RCT study}

The primary outcome measures will be:

1. Participants' knowledge about CFS and CFS interventions measured postintervention compared with baseline. Multiple choice and short answer questions, integrated with case vignettes, will test participants' knowledge about CFS symptoms, differential diagnosis, CFS management strategies and interventions (CBT and GET), and interventions for conditions that commonly arise secondary to fatigue (reduced mood and anxiety).

2. Participants' self-reported confidence in their knowledge of CFS and confidence in their clinical skills to implement evidence-based CFS interventions. This part of the questionnaire requires participants to rate their confidence in their knowledge and clinical skills related to CFS using a 5-point Likert Scale ('not at all confident', 'not very confident', 'somewhat confident', 'confident' and 'very confident').

These measures have been constructed by an expert research group consisting of physicians, exercise physiologists and clinical psychologists and designed to test knowledge across the range of allied health professions.

\section{Secondary outcome measures}

The secondary outcome measures will be:

1. The retention of knowledge about CFS and CFS interventions, and confidence of participants' in their clinical skills, across the follow-up period subsequent to the postintervention assessment. These data will be collected for the education group only.

2. Adherence to, and satisfaction with, the education activity. These data will be collected for the education group only. Intervention adherence will be tracked using in-built features of the online education platform, which will monitor: time spent in total on the activity, time spent on each module of the activity and engagement in integrated formative assessment tasks. In the postintervention 
Table 1 Intervention description using the Template for Intervention Description and Replication (TIDieR) checklist

Online education programme for allied health professionals on chronic fatigue

Brief name syndrome (CFS) and evidence-based CFS interventions

\begin{tabular}{|c|c|}
\hline Why & $\begin{array}{l}\text { The intervention has been designed based on a manual developed by the research group } \\
\text { that drew on Cochrane reviews of cognitive behaviour therapy (CBT) and graded exercise } \\
\text { therapy (GET) interventions for CFS. Additionally, a large review of internet-based education } \\
\text { programmes indicates online education interventions are as effective as traditional training } \\
\text { methods and have the advantage of being easily accessible. }^{19}\end{array}$ \\
\hline What materials & $\begin{array}{l}\text { The online education programme is presented as a mix of text, audiovisual resources } \\
\text { (including training videos) and interactive activities using the Smart Sparrow platform: https:/ } \\
\text { www.smartsparrow.com. }\end{array}$ \\
\hline What procedures & $\begin{array}{l}\text { A number of modules are presented in the online education programme as outlined below: } \\
\text { Introduction } \\
\text { - Defines chronic fatigue states (eg, postcancer fatigue) } \\
\text { - Fatigue assessment tools } \\
\text { Module 1: Psychoeducation } \\
\text { - Rationale underlying the intervention approach } \\
\text { Module 2: Activity pacing and GET } \\
\text {-Activity pacing } \\
\text { - Gradual progression of physical activities (GET) } \\
\text { Module 3: Interventions for sleep-wake cycle disturbance } \\
\text { - Symptoms of sleep-wake cycle disturbance } \\
\text { - Sleep hygiene and CBT interventions for sleep-wake cycle disturbance } \\
\text { Module 4: Interventions for neurocognitive disturbance } \\
\text {-Pacing of cognitive activities } \\
\text { - Gradual progression of cognitive activities (CET) } \\
\text { Module 5: Interventions for mood disturbance } \\
\text { - How to distinguish between depression and chronic fatigue states } \\
\text {-Psychoeducation for mood disturbance } \\
\text { - CBT intervention for mood disturbance } \\
\text { Module 6: Interventions for anxiety } \\
\text { - Anxiety symptoms } \\
\text { - CBT interventions for anxiety } \\
\text { Module 7: Interventions for coping } \\
\text { - Effective coping strategies }\end{array}$ \\
\hline Who provided & $\begin{array}{l}\text { The online education programme was designed and developed by five clinical psychologists } \\
\text { one research psychologist, five exercise physiologists and a medical specialist, all with } \\
\text { significant clinical experience in the management of people with chronic fatigue states. }\end{array}$ \\
\hline How & The online education programme is delivered individually as a self-paced online activity. \\
\hline Where & $\begin{array}{l}\text { Participants may access the online education programme wherever they have a device with } \\
\text { online access. }\end{array}$ \\
\hline When and how much & $\begin{array}{l}\text { Each participant will have access to the online education programme for a duration of } 4 \\
\text { weeks. The intervention is self-paced. The activity will collect data on how long participants } \\
\text { spend on each module and assessment activity. }\end{array}$ \\
\hline Tailoring & All participants will receive the same content. \\
\hline
\end{tabular}

CET, cognitive exercise therapy.

assessment, participants will be asked to rate their agreement with a number of statements related to their satisfaction with the online education activity. This will be done using a 5-point Likert Scale anchored at one end with 'strongly disagree' and at the other end with 'strongly agree'. Open-ended responses will also be collected.

\section{Cohort study outcomes}

Primary outcome measures are assessed at baseline and follow-up, and are:
1. Participants' self-reported success in the management of people with CFS. This part of the questionnaire requires participants to rate their level of success in the management of people with CFS using a 5-point Likert Scale, anchored at one end with 'completely unsuccessful' and at the other end with 'completely successful'.

2. Practice behaviours as evidenced by participants' self-reported proportion of clinical practice devoted to people with CFS. This part of the questionnaire requires the participant to indicate 
the percentage of their clinical practice that is devoted to management of people with CFS, for example the proportion of their clientele who have CFS, to determine levels of service provision.

\section{Analysis of outcomes}

Between-group differences at baseline (ie, education group versus wait-list control group) will be determined using independent t-tests for the knowledge assessment (primary outcome measure) and Mann-Whitney $\mathrm{U}$ tests for the Likert Scale ratings (secondary outcome measures). To determine if the group receiving the online education programme improves in knowledge and confidence related to the management of CFS, compared with the wait-list control group, an intention-to-treat analysis will be used. Group differences in change scores from baseline to postintervention will be analysed with independent t-tests for the knowledge assessment, and with Mann-Whitney U tests for the measure of clinician confidence. Short answer responses in the assessments will be graded in duplicate by blinded markers, using standardised answer criteria. Open-ended responses regarding participant satisfaction with the online education will be coded and analysed thematically using NVivo software. Descriptive statistics will be generated from Likert Scale responses to the questionnaire items regarding satisfaction with the intervention. Similarly, descriptive statistics will be generated for the total time spent on the online education programme, time spent on each module, total time spent on the integrated formative assessment tasks and responses on these tasks for each professional group. All statistical analyses will be conducted on deidentified data using SPSS software and the experimenter responsible for data analysis will be blinded to group allocation.

For the cohort study, retention of knowledge will be assessed using a dependent t-test comparing postintervention and follow-up assessment scores for the education group only. An intention-to-treat analysis will not be used in this instance so that retention of learning is not artificially inflated. To provide a preliminary indication of the impact of the intervention on clinical practice we will compare the change in measures of practice behaviour (ie, proportion of clinical practice devoted to the management of people with CFS) from baseline to follow-up in cohort study with the outcome measures for both groups combined. The range in proportion of clinical practice devoted to people with CFS at baseline for both groups will also be described to account for potential biases in sampling. This pragmatic approach should ensure a sufficient sample at follow-up.

\section{DISCUSSION}

Given the serious and debilitating nature of CFS and the absence of curative treatments, it is unfortunate that there has been limited uptake of evidence-based interventions aimed at managing symptoms and improving patient function. To our knowledge there have been no studies to date that have investigated the impact of an online education programme on clinician knowledge and skills related to CFS management by allied health professionals. This trial aims to evaluate if an online education programme improves clinicians' knowledge and confidence in the management of patients with CFS.

Reflective of the multidisciplinary nature of CBT/ GET interventions, the education intervention is being trialled across a range of allied health professions who will have different background experience with regard to the two major aspects of the intervention. Learning analytics within the intervention, and performance on specific sets of questions within the trial assessments, will identify the extent to which learner background has influenced performance. Coupled with feedback from the trial participants regarding their satisfaction and learning preferences, it is possible on the e-learning platform used in this trial to refine activities by incorporating adaptive features particular to groups or individuals to tailor content and feedback.

The cohort study component of the trial will generate preliminary evidence to determine if the intervention has an impact on clinical practice behaviours, which would also be amenable to further investigation using an optimised learning intervention. Given the possibility of convenience sampling of allied health professionals, further investigation regarding the efficacy of the intervention on a sample that have yet to formulate opinions regarding intervention for CFS would also be valuable (eg, implemented within a tertiary allied health training programme or mandated for all staff within a health professional service). The readily distributable online education intervention has the potential to improve the capacity of a range of allied health professionals to provide effective interventions for people with CFS.

Contributors BKB, AL, CXS and SHL conceived the study. SHL, BKB, CXS, SMC and $A L$ initiated the study design and $\mathrm{JC}$ and TB assisted with implementation. SHL, $B K B, C X S, S M C$ and $A L$ are grant holders. SHL and BKB are conducting the primary statistical analysis. All authors contributed to the refinement of the study protocol and approved the final manuscript.

Funding This work is supported by JJ Mason \& HS Williams Memorial Foundation (Grant number: MAS2015F040).

Competing interests None declared.

Ethics approval The University of New South Wales Human Research Ethics Committee.

Provenance and peer review Not commissioned; externally peer reviewed.

Open Access This is an Open Access article distributed in accordance with the Creative Commons Attribution Non Commercial (CC BY-NC 4.0) license, which permits others to distribute, remix, adapt, build upon this work non-commercially, and license their derivative works on different terms, provided the original work is properly cited and the use is non-commercial. See: http://creativecommons.org/ licenses/by-nc/4.0/

(C) Article author(s) (or their employer(s) unless otherwise stated in the text of the article) 2017. All rights reserved. No commercial use is permitted unless otherwise expressly granted.

\section{REFERENCES}

1. Fukuda K, Straus SE, Hickie I, et al. The chronic fatigue syndrome: a comprehensive approach to its definition and study. International 
chronic Fatigue syndrome Study Group. Ann Intern Med 1994;121:953-9.

2. Prins JB, van der Meer JW, Bleijenberg G. Chronic fatigue syndrome Lancet 2006;367:346-355.

3. Price JR, Mitchell E, Tidy E, et al. Cognitive behaviour therapy for chronic fatigue syndrome in adults. Cochrane Database Syst Rev 2008:CD001027.

4. Larun L, Brurberg KG, Odgaard-Jensen J, et al. Exercise therapy for chronic fatigue syndrome. Cochrane Database Syst Rev 2016;2:CD003200.

5. Castell BD, Kazantzis N, Moss-Morris RE. Cognitive behavioral therapy and graded exercise for chronic Fatigue syndrome: a MetaAnalysis. Clinical Psychology: Science and Practice 2011;18:311-24.

6. Vos-Vromans DC, Smeets RJ, Huijnen IP, et al. Multidisciplinary rehabilitation treatment versus cognitive behavioural therapy for patients with chronic fatigue syndrome: a randomized controlled trial. J Intern Med 2016;279:268-82.

7. Sandler CX, Hamilton BA, Horsfield SL, et al. Outcomes and predictors of response from an optimised, multidisciplinary intervention for chronic fatigue states. Intern Med J 2016;46:1421-9.

8. Dougall D, Johnson A, Goldsmith K, et al. Adverse events and deterioration reported by participants in the PACE trial of therapies for chronic fatigue syndrome. J Psychosom Res 2014;77:20-6.

9. Fernie BA, Murphy G, Wells A, et al. Treatment outcome and Metacognitive Change in CBT and GET for Chronic Fatigue syndrome. Behav Cogn Psychother 2016;44:397-409.

10. Lloyd AR, Meer JW. The long wait for a breakthrough in chronic fatigue syndrome. BMJ 2015;350:h2087.

11. Crawley E, Collin SM, White PD, et al. Treatment outcome in adults with chronic fatigue syndrome: a prospective study in England based on the CFS/ME National Outcomes Database. QJM 2013;106:555-65.

12. McDermott $C, A$ Haddabi $A$, Akagi $H$, et al. What is the current NHS service provision for patients severely affected by chronic fatigue syndrome/myalgic encephalomyelitis? A national scoping exercise. BMJ Open 2014:4:e005083.

13. Shafran R, Clark DM, Fairburn CG, et al. Mind the gap: improving the dissemination of CBT. Behav Res Ther 2009;47:902-9.

14. Weissman MM, Verdeli $\mathrm{H}$, Gameroff MJ, et al. National Survey of Psychotherapy Training in Psychiatry, Psychology, and Social Work. Arch Gen Psychiatry 2006;63:925.

15. Smith R, Smith R. Secondary Richard Smith: QMUL and King's college should release data from the PACE trial. http://blogs.bmj. com/bmj/2015/12/16/richard-smith-qmul-and-kings-college-shouldrelease-data-from-the-pace-trial/ (accessed Dec 2016).

16. Keech A, Sandler CX, Vollmer-Conna U, et al. Capturing the postexertional exacerbation of fatigue following physical and cognitive challenge in patients with chronic fatigue syndrome. J Psychosom Res 2015;79:537-49.

17. Sandler CX, Lloyd AR, Barry BK. Fatigue exacerbation by interval or continuous exercise in chronic fatigue syndrome. Med Sci Sports Exerc 2016;48:1875-85.

18. Goudsmit EM, Nijs J, Jason LA, et al. Pacing as a strategy to improve energy management in myalgic encephalomyelitis/ chronic fatigue syndrome: a consensus document. Disabil Rehabil 2012;34:1140-7.

19. Cook DA, Levinson AJ, Garside S, et al. Internet-based learning in the health professions: a meta-analysis. JAMA 2008;300:1181.

20. Cobb SC. Internet continuing education for health care professionals: an integrative review. J Contin Educ Health Prof 2004;24:171-80.

21. Wiborg JF, Knoop H, Wensing M, et al. Therapist effects and the dissemination of cognitive behavior therapy for chronic fatigue syndrome in community-based mental health care. Behav Res Ther 2012;50:393-6.

22. Fary RE, Slater H, Chua J, et al. Policy-Into-Practice for Rheumatoid arthritis: randomized Controlled Trial and Cohort Study of E-Learning targeting Improved Physiotherapy Management. Arthritis Care Res 2015;67:913-22.

23. Harvey LA, Glinsky JV, Lowe R, et al. A Massive Open Online Course for teaching physiotherapy students and physiotherapists about Spinal cord injuries. Spinal Cord 2014;52:911-918.

24. Tiedemann A, Sturnieks DL, Hill A-M, et al. Does a fall prevention educational programme improve knowledge and change exercise prescribing behaviour in health and exercise professionals? A study protocol for a randomised controlled trial. BMJ Open 2014;4:e007032.

25. Schulz KF, Altman DG, Moher D, Consort MD. CONSORT 2010 Statement: updated guidelines for reporting parallel group randomised trials. BMC Med 2010;8:18.

26. Chan AW, Tetzlaff JM, Gøtzsche PC, et al. SPIRIT 2013 explanation and elaboration: guidance for protocols of clinical trials. $B M J$ 2013;346:e7586.

27. Hoffmann TC, Glasziou PP, Boutron I, et al. Better reporting of interventions: template for intervention description and replication (TIDieR) checklist and guide. BMJ 2014;348:g1687. 